\title{
Realistic Models of Biological Motion
}

\author{
Imre Derényi and Tamás Vicsek \\ Department of Atomic Physics, Eötvös University \\ Budapest, Puskin u 5-7, 1088 Hungary
}

\begin{abstract}
The origin of biological motion can be traced back to the function of molecular motor proteins. Cytoplasmic dynein and kinesin transport organelles within our cells moving along a polymeric filament, the microtubule. The motion of the myosin molecules along the actin filaments is responsible for the contraction of our muscles. Recent experiments have been able to reveal some important features of the motion of individual motor proteins, and a new statistical physical description often referred to as "thermal ratchets" - has been developed for the description of motion of these molecules. In this approach the motors are considered as Brownian particles moving along one-dimensional periodic structures due to the effect of nonequilibrium fluctuations. Assuming specific types of interaction between the particles the models can be made more realistic. We have been able to give analytic solutions for our model of kinesin with elastically coupled Brownian heads and for the motion of the myosin filament where the motors are connected through a rigid backbone. Our theoretical predictions are in a very good agreement with the various experimental results. In addition, we have considered the effects arising as a result of interaction among a large number of molecular motors, leading to a number of novel cooperative transport phenomena.
\end{abstract}

\section{Introduction}

The most common and best known transport phenomena occur in systems in which there exist macroscopic driving forces (typically due to external fields or concentration gradients). However, recent theoretical studies have shown that far from equilibrium processes in structures possessing vectorial symmetry can bias thermal noise and induce macroscopic motion on the basis of purely microscopic effects [1-12]. This newly suggested mechanism is expected to be essential for the operation of molecular combustion motors that are responsible for many kinds of biological motion such as cellular transport or muscle contraction [13]. In these cases motor proteins (dynein, kinesin and

Preprint submitted to Elsevier Preprint 27 September 2018 
(a)

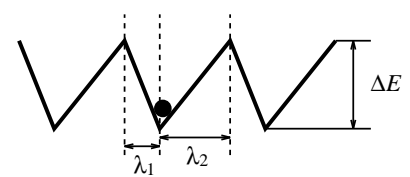

(b)

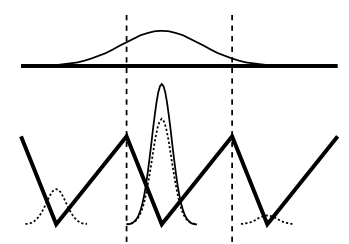

(c)

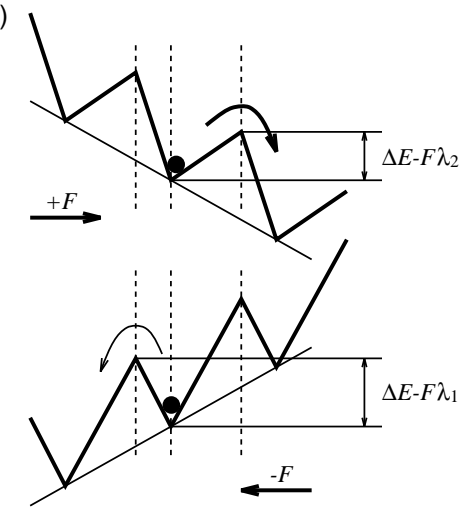

Fig. 1. The motion of a Brownian particle (a) in a sawtooth shaped potential due to the effect of (b) fluctuating potential and (c) fluctuating force.

myosin) convert the chemical energy stored in ATP molecules into mechanical work while moving along polymeric filaments (dynein and kinesin along microtubules, and myosin along actin filaments) [14]. A transport mechanism of this kind has also been experimentally demonstrated in simple physical systems [15-17] and can lead to new technological ideas such as constructing nano-scale devices or novel type of particle separators.

In the theoretical models - loosely termed "thermal ratchets" - molecular motors are considered as Brownian particles in an overdamped environment moving along one-dimensional asymmetric periodic potentials due to the effect of nonequilibrium fluctuations. For simplicity and illustration purposes let us choose the potential to be sawtooth shaped (Fig. 1a), and consider the two basic types of fluctuations: the fluctuating potential [1,3] (Fig. 1b) and the fluctuating force [2,3] (Fig. 1c).

In the case of the fluctuating potential the sawtooth potential is switched on and off repeatedly with appropriate switching rates. Fig. 1b illustrates the time evolution of the probability distribution of the position of the particle starting from one of the potential valleys. If the temperature $T$ is small enough the particle stays near the bottom of this valley until the potential is switched off. Then the particle starts to diffuse on the flat potential. Switching back to the sawtooth potential with the appropriate rate, the particle has a large probability to fall back to the original valley or to the neighboring valley to the left but only a small probability to fall back to the right one because of the asymmetry of the potential. Thus, a net motion to the left can be observed.

In the case of the fluctuating force $F(t)$ alternates between $+F$ and $-F$ with an appropriate characteristic frequency and with zero time average (Fig. 1c). When the force points to the right $(F(t)=+F)$, the probability of jumping to the right dominates the jumping events with the coefficient $\exp (-(\Delta E-$ $\left.\left.F \lambda_{2}\right) / k T\right)$ ). When the force is $-F$, the probability of jumping to the left dominates, but with the coefficient $\left.\exp \left(-\left(\Delta E-F \lambda_{1}\right) / k T\right)\right)$. And since $\lambda_{1}<$ 
$\lambda_{2}$, this fluctuating force results in a net motion to the right.

These simple ratchet models catch the basic mechanism of the operation of motor proteins, but they are too crude to be compared with real experiments. However, assuming specific types of interaction between the particles the models can be made more realistic. We call a model of biological motion realistic if it incorporates the relevant known features of motor proteins and leads to predictions fully consistent with experiments.

\section{Kinesin walk model}

Kinesin is a motor protein that moves large distances along microtubule filaments and transports organelles and vesicles inside the cytoplasm of eukaryotic cells. Native kinesin is a dimeric molecule with two globular $(\sim 9 \times 3 \times 3 \mathrm{~nm})$ heads. Microtubule is a long hollow tube the wall of which is made up of tubulin heterodimers arranged in 13 longitudinal rows called protofilaments. A tubulin heterodimer is $8 \mathrm{~nm}$ long and consists of two globular proteins about $4 \mathrm{~nm}$ in diameter: $\alpha$ - and $\beta$-tubulin. The dimers bind head-to-tail giving the polarity to the protofilaments. Electron micrograph measurements [18-21] indicate that kinesin heads can bind mainly to the $\beta$-tubulin.

Recent experimental studies in in vitro motility assays have revealed numerous important properties of kinesin movement, such as the unidirectionality of the motion, the force-velocity relationship, the time dependence of the displacement and the displacement variance [22-27].

There are two basic classes of possible models for the stepping of kinesin [28]. The first one is the "Long-Stride" model in which the heads are moving along a single protofilament. The two heads are displaced from each other by $8 \mathrm{~nm}$. During the stepping process the back head passes the bound front head advancing $16 \mathrm{~nm}$. Then the heads change their roles and a new step may take place. This model was studied in detail by Peskin and Oster [29]. The other possibility is the family of "Two-Step" models. These models naturally explain the measured low displacement variance because of the two sequential subprocesses: during one cycle one of the two heads takes a $8 \mathrm{~nm}$ step first then the other head steps. This kind of motion gives large stability to the protofilament tracking. The differences in the "Two-Step" models arise from the relative positions of the heads.

We propose a one-dimensional kinesin walk model [30] which describes the whole family of "Two-Step" models. Each of the two Brownian heads of the kinesin can move along its own one-dimensional periodic potential with period $L=8 \mathrm{~nm}$ in an overdamped environment. These potentials represent the inter- 


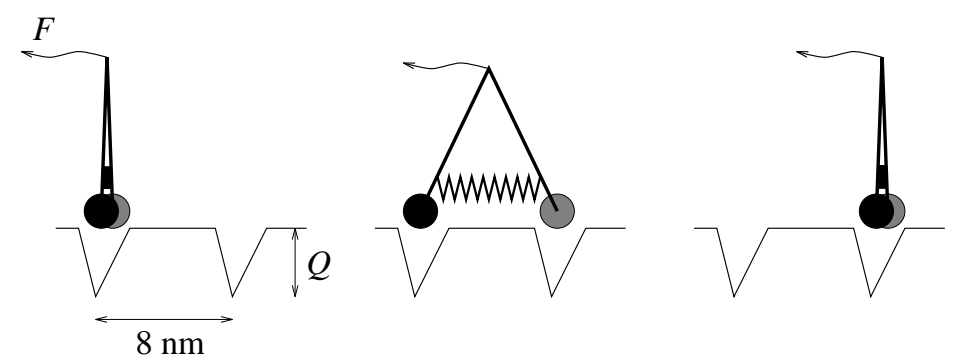

Fig. 2. Schematic picture of the potential and the subsequent steps of the kinesin molecule.

action with the protofilaments and the periods are the tubulin heterodimers. Each period has a deep potential valley corresponding to the binding site of the $\beta$-tubulin and the other parts of the potential are flat. Each valley has an asymmetric "V" shape (see Fig. 2): the slope in the backward direction is steeper and $0.5-1 \mathrm{~nm}$ long, while the other slope is $1.5-2 \mathrm{~nm}$ long. These ranges are in the order of the Debye length.

The heads are connected at a hinge, and a spring acts between them. The load force is joined to the heads through the hinge. At the beginning of the mechanochemical cycle both heads are sitting in their valleys waiting for an ATP molecule, and the spring is unstrained. Any configuration of the model is mathematically equivalent to a model in which the two potentials are identical, the heads are sitting in the same valley, and therefore the rest length of the spring is zero (Fig. 2). After one of the heads binds an ATP molecule, the hydrolysis of this ATP causes a conformational change in this head, more precisely, induces the head to take an $8 \mathrm{~nm}$ forward step. In the language of the model it means that the rest length of the spring changes from zero to $8 \mathrm{~nm}$ right after the hydrolysis. Then, as the first rate-limiting subprocess, the strained spring is trying to stretch pushing the head to the next valley. Reaching its new $8 \mathrm{~nm}$ rest length another conformational change occurs in the head as a consequence of the ADP release: the rest length of the spring changes back to zero quickly, then, as the second rate-limiting subprocess, the spring is trying to contract pulling forward the other head. Completing the contraction a next cycle can start waiting for a new ATP molecule. This picture is consistent with the scheme of Gilbert et al. [31] for the pathway of the kinesin ATPase, and with the very recent structural data for the kinesin's head $[32,33]$. The parameters of the model (viscous drag coefficient, stiffness of the spring, depth of the potential valleys) can be determined from well known experimental data, therefore, they can only be tuned in a rather restricted range.

Due to the asymmetry of the potential under low load force $F$ the kinesin molecule steps $8 \mathrm{~nm}$ forward during almost each mechanochemical cycle. But increasing the load the probability that the molecule remains on the same place or even takes a backward $8 \mathrm{~nm}$ step increases. The analytic solution of 


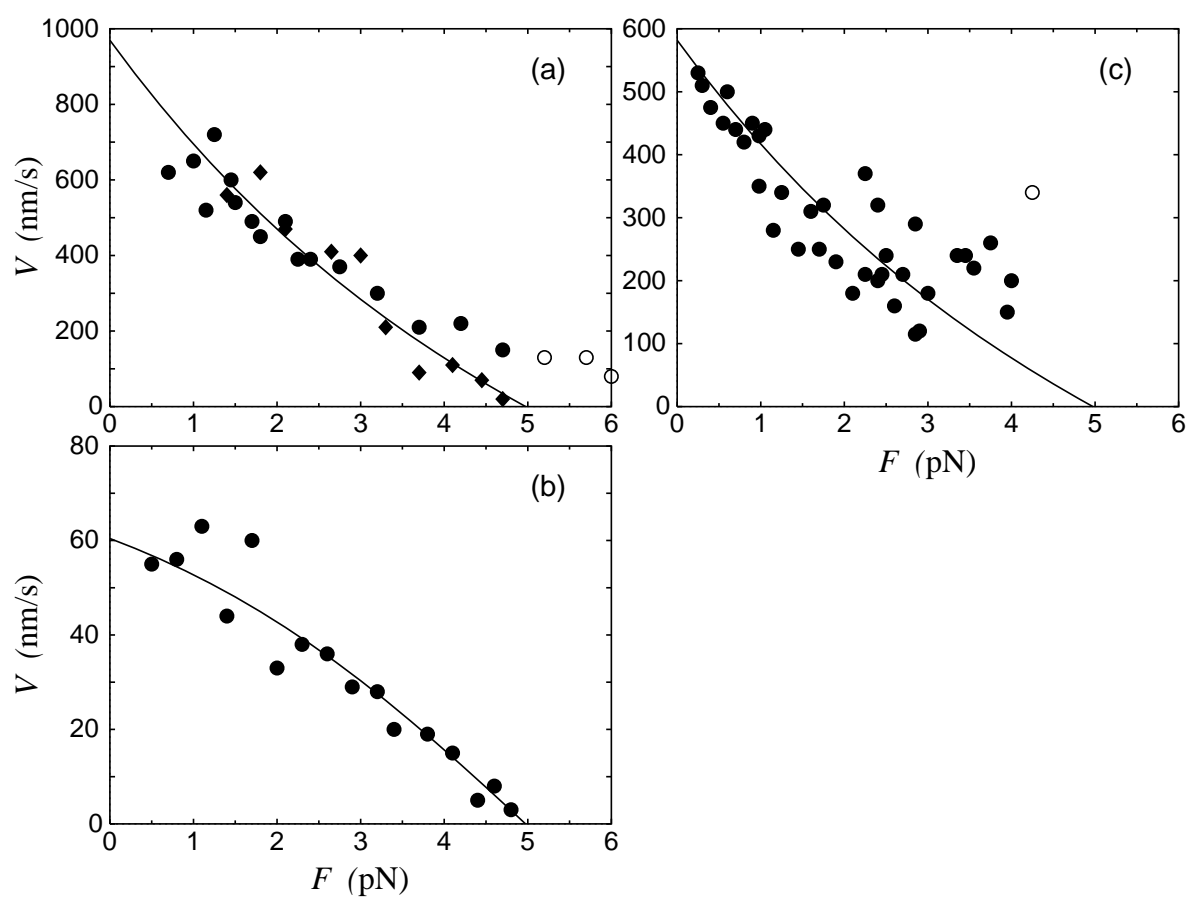

Fig. 3. The force-velocity curves for an individual kinesin molecule at saturating $(\gg 90 \mu \mathrm{M})$ ATP in (a) and (c); and at low $(\sim 10 \mu \mathrm{M})$ ATP concentration in (b). The experimental data (scattered symbols) are from Svoboda and Block [26] in (a) and (b); and from Hunt et al. [24] in (c). The open circles correspond to the simultaneous effect of multiple kinesin motors in the authors' interpretation [24,26]. The circles and diamonds in (a) mean two different set of the measured data.

the model [30] results in a very good fit to the measured mechanical properties of the kinesin walk (see, e.g., the force-velocity relationship in Fig. 3).

\section{Muscle contraction}

Muscle contraction results from a cyclic interaction between the actin filaments and their motor proteins - the myosin molecules. During the contraction the myosin cross-bridges extending from the thick myosin filaments attach to the binding sites of the thin actin filaments and exert force on them yielding a relative sliding of the actin and myosin filaments. The widely accepted scheme for the cross-bridge cycle [34,35], which was originally proposed by Lymn and Taylor [36], is 


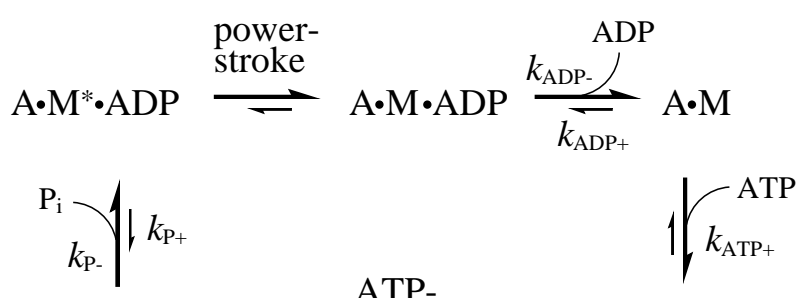

ATP-

$(\mathrm{A}) \cdot \mathrm{M} \cdot \mathrm{ADP} \cdot \mathrm{P}_{\mathrm{i}}=(\mathrm{A}) \cdot \mathrm{M} \cdot \mathrm{ATP}$

Initially, the myosin cross-bridge is in a weakly bound, non-force-producing $(\mathrm{A}) \cdot \mathrm{M} \cdot \mathrm{ADP} \cdot \mathrm{P}_{\mathrm{i}}$ state. $\mathrm{A}$ and $\mathrm{M}$ denote actin and myosin respectively, and (A) indicates that myosin is weakly bound to actin. Releasing the phosphate $\mathrm{P}_{\mathrm{i}}$ the cross-bridge gets into a strongly bound, force-producing $\mathrm{A} \cdot \mathrm{M}^{*} \cdot \mathrm{ADP}$ state. Then the cross-bridge executes a power stroke (see Fig. 4a) producing mechanical force on actin, a conformational change in myosin, and a relative sliding between adjacent actin and myosin filaments. This new $\mathrm{A} \cdot \mathrm{M} \cdot \mathrm{ADP}$ state cannot readily bind $\mathrm{P}_{\mathrm{i}}$ any more, in contrast to the preceding one. At the end of the power-stroke, when continued attachment would resist useful work, ADP is released with transition to the $\mathrm{A} \cdot \mathrm{M}$ state, which is still strongly bound. ADP may then rebind, resulting in continued counter-productive attachment, or ATP may bind, resulting in rapid cross-bridge detachment. The hydrolysis step occurs in this weakly bound $(\mathrm{A}) \cdot \mathrm{M} \cdot \mathrm{ATP}$ state resulting the $(\mathrm{A}) \cdot \mathrm{M} \cdot \mathrm{ADP} \cdot \mathrm{P}_{\mathrm{i}}$ cross-bridge state, which is free to participate in a new working cycle. The conformations of the cross-bridge states was determined by EPR spectroscopy [37].

One of the most important problems is to derive the macroscopic mechanical properties of muscle contraction from the microscopic biochemical steps of the cross-bridge interaction. The first and very influential analysis was presented by Huxley [38]. With his original two-state model he was able to reproduce much of the experimental data available at that time. Since then the models have become finer and more complicated to fit the increasing number of experimental data [39-44]. The general disadvantages of these models are that (i) they incorporate several functions among the other fitting parameters, (ii) and they can be solved only numerically.

We propose a simple mechanochemical model [45] for the cross-bridge interaction that is free from the above mentioned shortcomings. Our four-state model (i) involves the relevant kinetic data and other microscopic parameters, (ii) and provides simple analytic solutions for the mechanical properties of muscle contraction. These analytic results fit numerous experimental data extremely well and make clear the relationship between the microscopic and macroscopic parameters. We would like to point out that analytic solutions are extremely useful if one would like to go beyond merely giving a phenomenological description of a biological phenomenon, since the structure of the analytic expressions explicitly relating the relevant factors provides a unique insight into the role 
of these factors. The model is based on the following assumptions:

(i) The distance between two neighboring binding sites on the actin filament is $d \approx 37 \mathrm{~nm}$ being the half period of the double helix of the actin filament. The position of the actin filament is fixed while the myosin filament passes by it with a velocity $V$ from left to right. Since the spacing of the myosin heads and the actin binding sites are incommensurate, the distances between the myosin cross-bridges and their nearest binding sites are uniformly distributed within the range $[-d / 2, d / 2]$. Thus, the macroscopic properties of muscle contraction can be calculated as a spatial average for the position of one cross-bridge (more precisely its S1-S2 junction, denoted by $x$ ) over this interval.

(ii) Initially the myosin cross-bridge is in a weakly bound, non-force-producing state that can be represented by a flat potential (Fig. 4b). When the crossbridge gets into the vicinity $-l \leq x \leq-l+\delta$ of the actin binding site, phosphate can be released forming a stereospecific, strongly bound, forceproducing state. $l$ denotes the power-stroke working distance for which the most accurate value $l \approx 11 \mathrm{~nm}$ was measured by using optical tweezers [46]. This is also consistent with the structural studies of myosin $[47,48] . \delta$ is the composition of the Debye length and the rotational freedom of the myosin head, and can be well approximated by a few $\mathrm{nm}$. The force produced by this state in the power-stroke region $(-l \leq x \leq 0)$ is about $f \approx 4 \mathrm{pN}$ which was also measured by Finer et al. [46]. Finishing the power-stroke continued attachment resists useful work, i.e., exhibits a negative force $-f^{\prime}$ in this $d r a g$ -

(a)

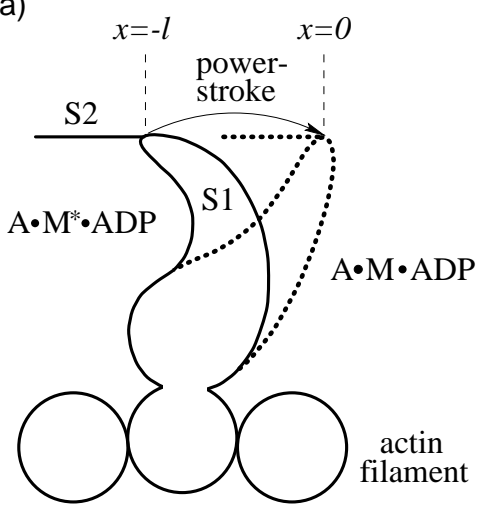

(b)

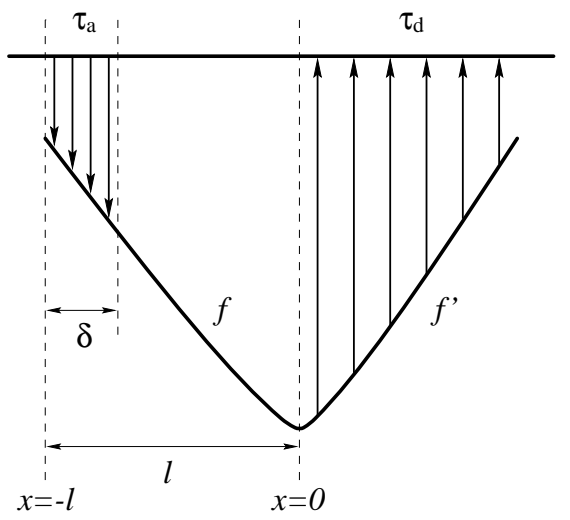

Fig. 4. Cartoon (a) shows the conformational change in myosin head during the power-stroke. On the schematic picture (b) the flat and "V" shaped potentials represent the weakly and strongly bound states of the myosin cross-bridge. The slopes of the "V" shaped potential are $f$ and $f^{\prime}$ that correspond to the force produced by the myosin head in the power-stroke and in the drag-stroke region respectively. Transition from the weakly bound state to the strongly bound state can occur in the $-l<x<-l+\delta$ region with characteristic time $\tau_{\mathrm{a}}$ and transition from the strongly bound state to the weakly bound state in the $0<x$ with characteristic time $\tau_{\mathrm{d}}$. These time constants are determined by the kinetic rates and the substrate concentrations. 


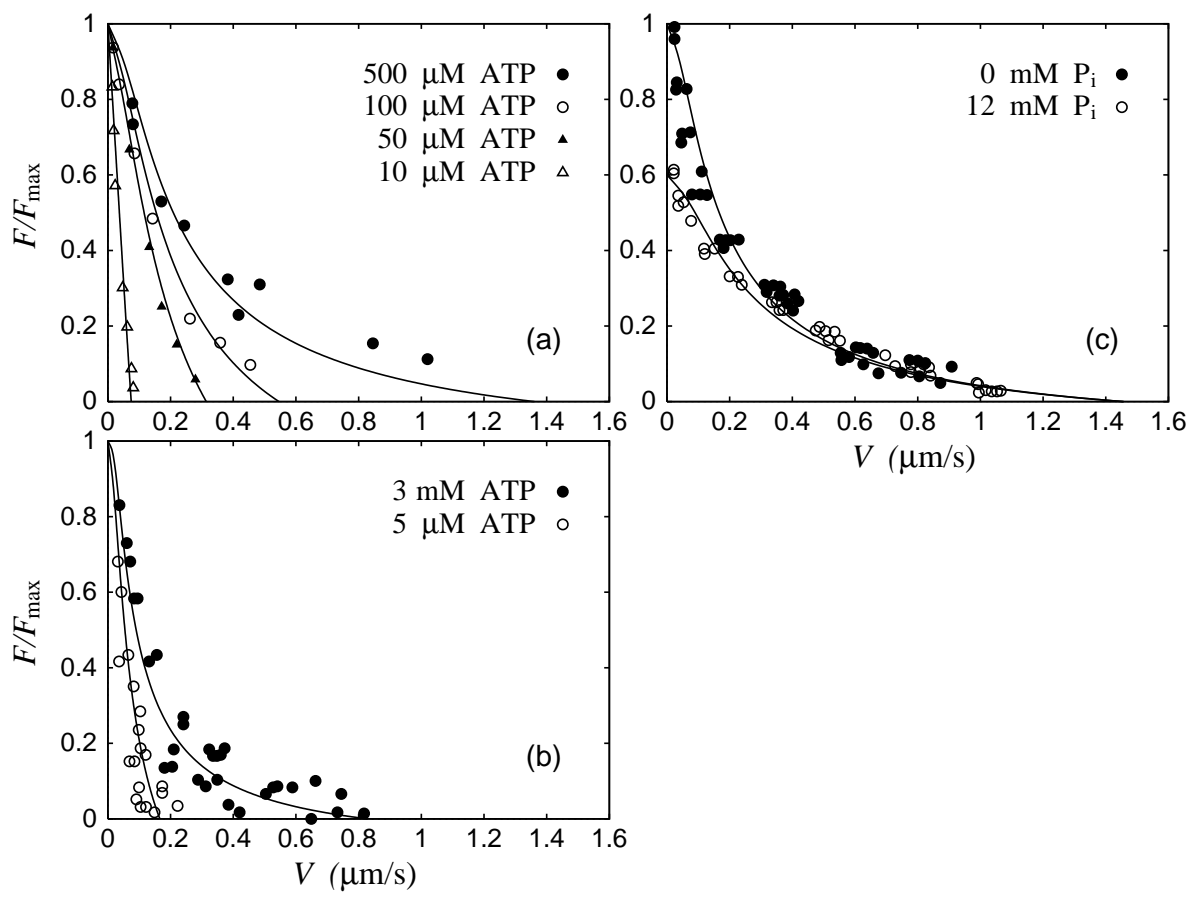

Fig. 5. The force-velocity relationship of the contracting muscle in various situations: rabbit psoas muscle [49] in (a) and rabbit semimembranosus muscle [50] in (b) for different ATP concentrations in the absence of ADP and $\mathrm{P}_{\mathrm{i}}$; and rabbit psoas muscle [51] in (c) for two different $P_{i}$ concentrations in the absence of ADP at saturating $(4 \mathrm{mM})$ ATP. The scattered symbols are experimental data and the solid lines are fits of the model.

stroke region $(0<x)$. $f^{\prime}$ must be close to $f$, but not necessarily equals to it. Altogether, the strongly bound state can be represented by a "V" shaped potential shown in Fig. $4 \mathrm{~b}$.

(iii) In the drag-stroke region $(0<x)$ ADP can be released with transition to another strongly bound state which is supposed to have similar properties, and can also be represented by the same "V" shaped potential. Then ATP can bind resulting in rapid cross-bridge detachment. And finally a hydrolysis step occurs in this weakly bound state which is represented by the flat potential.

The analytic solution of this model [45] agrees with numerous experimental results very well: Fig. 5 shows some fits to the measured force-velocity [49-51]. The well known empirical Hill equation [52] also gives a good fit, although, its relationship to the biochemical level is unknown. The model results in a Michaelis-Menten-like saturation behavior for the maximum shortening velocity [49-51,53,54] and the ATPase activity [55] as a function of the ATP concentration, which is also in good agreement with the experimental results. Further features of our model are also consistent with the experiments [56-58]. In addition, the model gives a prediction for the proportion of the attached cross-bridges. This is very difficult to measure and the recent experimental 
results are contradictory.

\section{Cooperative motors}

From the previous sections it has turned out that specific interaction between the motors has significant effect on their motion. Elastic interaction between two [59] or more [60] particles and a general model of rigidly coupled motors [61] has been recently investigated that revealed numerous peculiar collective phenomena.

Since in real biological [62] and physical [15] systems a large number of free motors can move along the same structure we have investigated the collective motion of finite sized Brownian particles in a one-dimensional sawtooth shaped potential for both the fluctuating force [63] and the fluctuating barrier [64]. The interaction between the particles is supposed to be a simple hard core repulsion, thus the particles can be considered as hard rods. This type of interaction means that during the motion the particles are not allowed to overlap.

Analytical treatment of some special sets of parameters and computer simulations have revealed that this simple system exhibits many interesting collective transport properties, e.g.:

(i) Increasing the density of the particles (defined as the number of particles $\times$ the size of the particles / the system size) from 0 to 1 the direction of their motion can change its sign several times.

(ii) Close to the maximal density the the average velocity shows a very strong and complex dependence on the size of the particles.

\section{References}

[1] A. Ajdari and J. Prost. C. R. Acad. Sci. Paris, 315:1635, 1992.

[2] M. O. Magnasco. Phys. Rev. Lett., 71:1477, 1993.

[3] R. D. Astumian and M. Bier. Phys. Rev. Lett., 72:1766, 1994.

[4] C. R. Doering, W. Horsthemke, and J. Riordan. Phys. Rev. Lett., 72:2984, 1994.

[5] J. Prost, J.-F. Chauwin, L. Peliti, and A. Ajdari. Phys. Rev. Lett., 72:2652, 1994.

[6] A. Ajdari, D. Mukamel, L. Peliti, and J. Prost. J. Phys. I France, 4:1551, 1994. 
[7] C. S. Peskin, G. B. Ermentrout, and G. F. Oster. In V. C. Mow et al., editors, Cell Mechanics and Cellular Engineering. Springer-Verlag, New York, 1994.

[8] R. Bartussek, P. Hänggi, and J. G. Kissner. Europhys. Lett., 28:459, 1994.

[9] M. M. Millonas and D. I. Dykman. Phys. Lett. A, 185:65, 1994.

[10] M. M. Millonas. Phys. Rev. Lett., 74:10, 1995.

[11] R. D. Astumian and M. Bier. Biophys. J., 70:637, 1996.

[12] P. Hänggi and R. Bartussek. In J. Parisi, S. C. Müller, and W. Zimmermann, editors, Nonlinear Physics of Complex Systems, Lecture Notes in Physics, volume 467. Springer, Berlin, 1996.

[13] B. G. Levi. Phys. Today, 48(4):17, 1995.

[14] J. Darnell, H. Lodish, and D. Baltimore. Molecular Cell Biology. Scientific American Books, New York, 1990.

[15] J. Rousselet, L. Salome, A. Ajdari, and J. Prost. Nature, 370:446, 1994.

[16] L. P. Faucheux, L. S. Bourdieu, P. D. Kaplan, and A. J. Libchaber. Phys. Rev. Lett., 74:1504, 1995.

[17] L. P. Faucheux and A. J. Libchaber. J. Chem. Soc. Faraday. Trans., 91:1363, 1995.

[18] Y.-H. Song and E. Mandelkow. Proc. Natl. Acad. Sci. USA, 90:1671, 1993.

[19] A. Hoenger, E. P. Sablin, R. D. Vale, R. J. Fletterick, and R. A. Milligan. Nature, 376:271, 1995.

[20] M. Kikkawa, T. Ishikawa, T. Wakabayashi, and N. Hirokawa. Nature, 376:274, 1995.

[21] K. Hirose, A. Lockhart, R. A. Cross, and L. A. Amos. Nature, 376:277, 1995.

[22] R. D. Vale, T. S. Reese, and M. P. Sheetz. Cell, 42:39, 1985.

[23] S. Ray, E. Meyhöfer, R. A. Milligan, and J. Howard. J. Cell. Biol., 121:1083, 1993.

[24] A. J. Hunt, F. Gittes, and J. Howard. Biophys J., 67:766, 1994.

[25] K. Svoboda, C. F. Schmidt, B. J. Schnapp, and S. M. Block. Nature, 365:721, 1993.

[26] K. Svoboda and S. M. Block. Cell, 77:773, 1994.

[27] K. Svoboda, P. P. Mitra, and S. M. Block. Proc. Natl. Acad. Sci. USA, 91:11782, 1994.

[28] S. M. Block and K. Svoboda. Biophys J., 68:230s, 1995.

[29] C. S. Peskin and G. Oster. Biophys J., 68:202s, 1995. 
[30] I. Derényi and T. Vicsek. Proc. Natl. Acad. Sci. USA, 93:6775, 1996.

[31] S. P. Gilbert, M. R. Webb, M. Brune, and K. A. Johnson. Nature, 373:671, 1995.

[32] F. J. Kull, E. P. Sablin, R. Lau, R. J. Fletterick, and R. D. Vale. Nature, 380:550, 1996.

[33] E. P. Sablin, F. J. Kull, R. Cooke, R. D. Vale, and R. J. Fletterick. Nature, 380:555, 1996.

[34] Y. E. Goldman. Ann. Rev. Physiol., 49:629, 1987.

[35] C. R. Bagshaw. Muscle Contraction. Chapman \& Hall, London, 1993.

[36] R. W. Lymn and E. W. Taylor. Biochemistry, 10:4617, 1971.

[37] E. M. Ostap and D. D. Thomas. Biophys. J., 68:335s, 1995.

[38] A. F. Huxley. Prog. Biophys., 7:255, 1957.

[39] T. L. Hill. Prog. Biophys. Mol. Biol., 28:267, 1974.

[40] E. Eisenberg, T. L. Hill, and Y. Chen. Biophys. J., 29:195, 1980.

[41] E. Pate and R. Cooke. J. Muscle Res. Cell Motil., 10:181, 1989.

[42] E. Pate, H. White, and R. Cooke. Proc. Natl. Acad. Sci. USA, 90:2451, 1993.

[43] N. J. Córdova, B. Ermentrout, and G. F. Oster. Proc. Natl. Acad. Sci. USA, 89:339, 1992.

[44] S. Leibler and D. A. Huse. J. Cell. Biol., 121:1357, 1993.

[45] I. Derényi and T. Vicsek. Biophys. J., 1997. (submitted).

[46] J. T. Finer, R. M. Simmons, and J. A. Spudich. Nature, 368:113, 1994.

[47] I. Rayment, H. M. Holden, M. Whittaker, C. B. Yohn, M. Lorenz, K. C. Holmes, and R. A. Milligan. Science, 261:58, 1993.

[48] A. J. Fisher, C. A. Smith, J. Thoden, R. Smith, K. Sutoh, H. M. Holden, and I. Rayment. Biophys. J., 68:19s, 1995.

[49] R. Cooke and W. Bialek. Biophys. J., 28:241, 1979.

[50] E. Pate, M. Lin, K. Franks-Skiba, and R. Cooke. Am. J. Physiol., 262:C1039, 1992.

[51] R. Cooke and E. Pate. Biophys. J., 48:789, 1985.

[52] A. V. Hill. Proc. R. Soc. Lond., B126:136, 1938.

[53] S. J. Kron and J. A. Spudich. Proc. Natl. Acad. Sci. USA, 83:6272, 1986.

[54] Y. Harada, A. Noguchi, A. Kishino, and T. Yanagida. Nature, 326:805, 1987. 
[55] J. Sleep and H. Glyn. Biochemistry, 25:1149, 1986.

[56] B. Katz. J. Physiol., 96:45, 1939.

[57] A. F. Huxley and R. M. Simmons. Nature, 233:533, 1971.

[58] K. A. P. Edman and J. C. Hwang. J. Physiol., 269:255, 1977.

[59] A. Ajdari. J. Phys. I France, 4:1577, 1994.

[60] Z. Csahók, F. Family, and T. Vicsek. Phys. Rev. E, 55:5179, 1997.

[61] F. Jülicher and J. Prost. Phys. Rev. Lett., 75:2618, 1995.

[62] A. Ashkin, K. Schütze, J. M. Dziedzic, U. Euteneuer, and M. Schliwa. Nature, 348:346, 1990.

[63] I. Derényi and T. Vicsek. Phys. Rev. Lett., 75:374, 1995.

[64] I. Derényi and A. Ajdari. Phys. Rev. E, 54:R5, 1996. 